\title{
Study of the emissivity of rough surfaces periodic using the method of coupled waves analysis (CWA) compared with method of geometrical optics approximation (GOA)
}

\section{Taoufik Ghabara}

Department of Physics-Faculty of Science and Arts at Al-Rass-Qassim University, Qassim, Kingdom of Saudi Arabia; Taoufik.ghabara@ipein.rnu.tn

Received 23 June 2010; revised 25 July 2010; accepted 28 July 2010.

\section{ABSTRACT}

We present in this paper a numerical study of the validity limit of the geometrical optics approximation compared with a differential method which is established according to rigorous formalisms based on the electromagnetic theory. The precedent studies show that this method is adapted to the study of diffraction by periodic rough surfaces. We determine by two methods the emissivity of gold and tungsten for surfaces with a rectangular or sinusoidal profile, for a wavelength equal to 0.55 microns. The monochromatic directional emissivity of these surfaces clearly depends on the angle of incidence, the surface profile, height, period and the nature of the material. We perform our calculations by a method of coupled wave analysis (CWA) and a geometric optics method (GOA). The latter method is theoretically valid only when the dimensions of the cavities are very large compared to the wavelength, while the CWA is theoretically correct whatever these dimensions. The main purpose of this work is to investigate the validity limit of GOA compared with CWA. The obtained results for a fixed height of the grating, allowed us to delimit the validity domain of the optic geometrical approximation for the treated cases. Finally, the agreement between the emissivity calculated by the differential method and that given on the basis of the homogenization theory is satisfactory when the period is much smaller than the wavelength.

Keywords: Periodic Roughness; Differential Method (CWA); Geometric Optics Approximation (GOA); Homogenization Theory; Emissivity.

\section{INTRODUCTION}

The theoretical or experimental determination of radiative properties of rough surfaces is the subject of several researches. The modelling of directional monochromatic emissivity of a rough surface remain a subject of theoretical, experimental and numerical researches $[1,2]$.

These parameters are involved in several application areas ranging from the calculation of energy exchange by radiation in the design of selective rough surfaces, in addition to current applications on the semiconductor industry [3] and the Solar energy [4].

In this context two methods are presented, one exact and based on electromagnetic theory, while the second is approximate and based on geometrical optics. Among the exact methods, we mention the integral and differential methods.

While the integral methods are more effective to study the scattering of electromagnetic waves by rough surfaces $[5,6]$, the differential methods seem best suited to solving the same problem with periodic rough surfaces.

These methods established by the rigorous formalisms based on electromagnetic theory are known under the name of coupled wave analysis (coupled wave analysis CWA) and they are first applied to planar gratings [7-10] and also at deep profile periodic gratings dielectrics or conductors [11-14].

In parallel, various versions of algorithms were proposed. However, some of the solution algorithms are unstable and to remedy this, several numerical algorithms are developed to solve this problem for very deep gratings [15-17].

The approximate method adopted is based on optic geometrical approximation and it is only valid in the case of macro-roughness [18-22]. As soon as the dimensions of the roughness become comparable to or less than the wavelength, this method can be relatively chosen. 
This work has established the region of validity between the geometric optics approximation (GOA) and differential method (CWA) especially in the cases of rectangular and sinusoidal surfaces with a conductivity (gold and tungsten) lying in the visible. We have analysed the different physical phenomena depending on the period and the angles of incidence. We also study the radiative of the rough surface when its period is very small compared to the wavelength. The wavy portion is equivalent in these conditions to a layering of effective indices determined using the effective medium theory [23-25].

\section{DERIVATION OF THE EMISSIVITY USING DIFFERENTIAL METHOD (CWA)}

\subsection{Geometry}

In this study, we have considered a grating with rectangular or sinusoidal grooves. An electromagnetic wave obliquely incident upon the grating produces both reflected and transmitted waves, as it is shown in the Figure 1. Region 1 is a homogeneous dielectric with a relative permittivity of $\varepsilon_{1}$. Likewise, region 3 is homogeneous with a complex permittivity $\varepsilon_{3}$.

Region 2 (the grating region) consists of periodic distribution of both types of materiel. In this paper, for simplicity, we have assumed that the incident light has transverse electric (TE) polarization.

\subsection{Theory of Differential Method}

In the present analysis, the differential method CWA (coupled Wave Analysis) is adapted to the exact electromagnetic boundary value problem associated with dielectric and metallic miro-rough periodic surfaces $[13,14]$.

We consider an incident plane wave of wave vector, located in the plane $(\mathrm{xOz})$, in the case of TE polarization. The problem is to determine the amplitudes of reflected

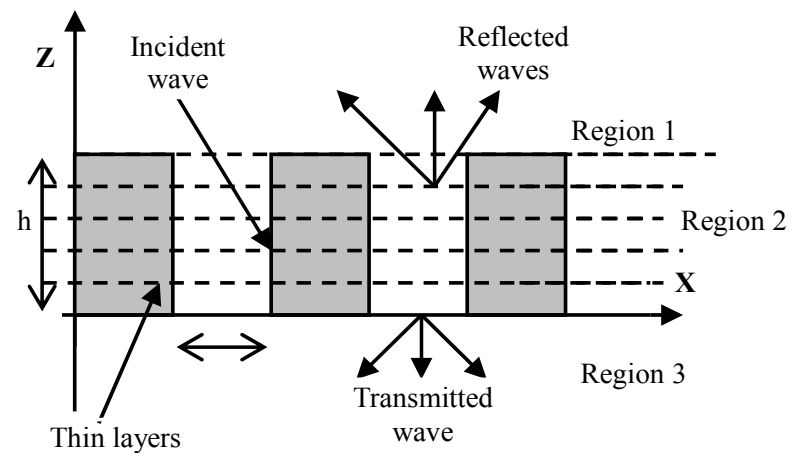

Figure 1. Surface with rectangular grooves. and transmitted fields. The total electric field in Region 1 is the sum of the incident waves and reflected waves. The amplitude of this field is given by:

$$
E_{1}=\exp \left(-j \vec{k}_{1} \cdot \vec{r}\right)+\sum_{i=-\infty}^{+\infty} R_{i} \exp \left(-j \vec{k}_{1 i} \cdot \vec{r}\right),
$$

$k_{1}$ is the incident wave vector; $R_{i}$ is the ith amplitude of the reflected wave in region 1 . The diffracted field in region 3 is given by:

$$
E_{3}=\sum_{i=-\infty}^{+\infty} T_{i} \exp \left(-j \vec{k}_{3 i} \cdot(\vec{r}-h \cdot \vec{z})\right),
$$

where $T_{i}$ is the amplitude of the ith transmitted wave in region 3 in this method, a surface relief grating (region 2) is divided in $\mathrm{M}$ thin layers similar to planar grating perpendicular to the axis $(\mathrm{Oz})$ (Figure 1), then applying the method of coupled waves at each layer [10]. The permittivity of the kth layer is a periodic function by (Ox), who's Fourier series expansion can be expressed by:

$$
\varepsilon_{k}\left(x, z_{k}\right)=\sum_{1=-\infty}^{+\infty} \tilde{\varepsilon}_{l, k} \exp (j 1 k x),
$$

where $k$ is the grating vector $(k=2 \pi / d), \tilde{\varepsilon}_{l, k}$ are the complex coefficients of Fourier series expansion. The field in each sub-layer is given by:

$$
E_{2, k}=\sum_{i=-\infty}^{+\infty} S_{i, k}(z) \exp \left(-j \vec{\sigma}_{i, k} \cdot \vec{r}\right),
$$

where $S_{i, k}$ is the amplitude of the diffracted field of order $i$ and $\vec{\sigma}_{i, k}$ is the diffracted wave vector in the interior of the kth layer.

In region 2 we have introduced the fundamental wave equation in TE polarization:

$$
\nabla^{2} E_{2, k}+k^{2} \varepsilon_{k}\left(x, z_{k}\right) E_{2, k}=0
$$

By replacing the field expansions (3) and (4) in the wave equation, we have obtained a differential system with constant coefficients according to each thin grating $k$ :

$$
\begin{aligned}
& \frac{d^{2} S_{i, k}(z)}{d z^{2}}-2 j\left(k_{2, k}^{2}-k_{1}^{2} \sin \theta^{2}\right)^{1 / 2} \frac{d S_{i, k}(z)}{d z} \\
& +K^{2} i(m-i) S_{i, k}(z)+k^{2} \sum_{i \neq p} \tilde{\varepsilon}_{p, k} S_{i-p, k}=0
\end{aligned}
$$

where: $m=2 d\left(\varepsilon_{1}^{1 / 2}\right) \sin \theta / \lambda$

\subsection{Method Resolution}

The transformation: $S_{1, i, k}(z)=S_{i, k}(z)$ and $S_{2, i, k}(z)$ $=d S_{i, k}(z) / d z$, leads to the linear system of differential equations of first order, is written in matrix form as follows: 


$$
\left[\left(S^{\prime}\right)_{l, p, k}\right]=[b] \times\left[(S)_{l, q, k}\right]
$$

For $l=1,2$ and $S^{\prime}=d S / d z$.

The matrix coefficients are given in [13] $(p=1, N$ et $q$ $=1, N)$. A solution of system (7) is given by:

$$
S_{p^{\prime}, k}=\sum_{q^{\prime}=1}^{2 N} C_{q^{\prime}, k} W_{p^{\prime}, q^{\prime}, k} \exp \left(\lambda_{q^{\prime}, k} \cdot z\right)
$$

where $\lambda_{q^{\prime}, k}$ is the eigenvalues and $W_{p^{\prime}, q^{\prime}, k}$ is the eigenvectors of the matrix. $C_{q^{\prime}, k}$ are the constants to be determined, first using the boundary conditions.

\subsection{Derivation of the Emissivity Using Differential Method (CWA)}

For solving the problem without numerical difficulties, we have adopted the stable algorithm presented by M.G Moharam [14] for transverse electric polarization (TE).

When the reflected $R_{i}$ and transmitted $T_{i}$ field complexes amplitudes are known, the diffraction efficiencies (ratio of diffracted intensity to input intensity) may be directly determined. Then the diffraction efficiencies in region 1 and 3 are:

$$
\begin{aligned}
& D E_{1 i}=\operatorname{Re}\left[\left(\vec{k}_{1 i} \cdot \vec{z}\right) /\left(\vec{k}_{10} \cdot \vec{z}\right)\right] R_{i} R_{i}^{*} \\
& D E_{3 i}=\operatorname{Re}\left[\left(\vec{k}_{3 i} \cdot \vec{z}\right) /\left(\vec{k}_{10} \cdot \vec{z}\right)\right] T_{i} T_{i}^{*}
\end{aligned}
$$

The emissivity is given by the following relation [26]:

$$
\varepsilon_{\lambda}(\theta)=1-\sum_{i=0} D E_{1 i}
$$

\section{DERIVATION OF THE EMISSIVITY USING GEOMETRICAL OPTICS APPROXIMATION (GOA)}

The method of geometrical optics is one of approximate methods of calculating the emissivity rough surfaces. It is based on the concept of light ray, and we use the notions of classical geometry or analytical related to Snell's law to determine the path of the incident beam inside the cavity. We adopt the approach that the principle is to consider a beam of parallel rays incident on the cavity at an angle of incidence and to determine the part absorbed [31-32].

The emissivity derived from geometrical optics is equal to:

$$
\varepsilon_{\lambda}\left(\theta, X_{s_{j}}\right)=1-\prod_{i=1}^{n} \rho_{\lambda}\left(\phi_{i}\right)
$$

where $\phi_{i}$ designates the local reflection angle at the interaction point $\mathrm{S}_{\mathrm{i}}$ (Figure 2) and $\rho_{\lambda}\left(\phi_{i}\right)$ is the Fresnel reflection factor.

In the case of sinusoidal cavities (Figure 2), the direc- tional emissivity is simply the average of all positions $X_{s_{j}}$, and can be written as:

$$
\varepsilon_{\lambda}(\theta)=\frac{1}{d} \int_{0}^{d} \varepsilon_{\lambda}\left(\theta, X_{s_{j}}\right) d X
$$

where $d$ is the grating period.

For rectangular cavities, determining the number of impact points inside the cavity and the local angles of incidence or reflection is done using a geometric study to show that the number of reflections $N_{j}$ inside the cavity is related to the integer part of quantity:

$$
2 \frac{h}{d} \tan \theta-2\left(1-\frac{X_{s_{j}}}{d}\right), X_{s_{j}} \in\left[\frac{d}{2}, d\right]
$$

The directional emissivity is given by:

$$
\varepsilon_{\lambda}(\theta)=1-\rho(\theta)\left[\rho\left(\frac{\pi}{2}-\theta\right)\right]^{N_{j}-1}
$$

\section{CONCEPT OF HOMOGENIZATION}

For gratings with a period much smaller than the wavelength, the roughness essentially behaves as a transition layer with a gradient of the optical index. The emissivity is given by the following relation [26]:

$$
\varepsilon_{\lambda}(\theta)=1-|r|^{2}
$$

where $r$ is the ratio of complex amplitude of reflected field and the complex amplitude of the incident field.

\section{RESULTS AND DISCUSSIONS}

We present results of our numerical calculations of the emissivity of gratings with a rectangular or sinusoidal

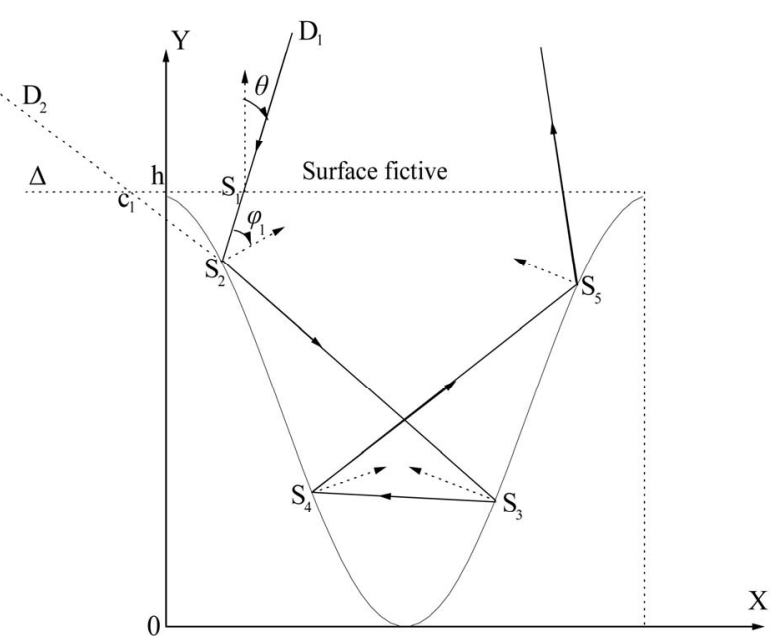

Figure 2. Reflections successive associated with an incident ray of the cavity sinusoidal. $\mathrm{D}_{1}$ : incident ray; $\mathrm{S}_{\mathrm{i}}$ : interaction points; $\theta$ incidence angle; $\varphi_{1}$ : local reflection angle. 
grooves for height $\mathrm{h}$ and period $\mathrm{d}$. We associate these surfaces defined by the angle: $\tan \beta=2 h / d$. Note that when $d / \lambda$ varies from 0.05 to 12.8 angle, $\beta$ varies from $75.96^{\circ}$ to $0.89^{\circ}$ for $h=0.1 \lambda$ and from $88.56^{\circ}$ to $8.88^{\circ}$ for $h=1 \lambda$.

The grooves are composed of materials of gold $(\mathrm{Au})$ and tungsten $(\mathrm{W})$, of respective refractive indexes $\mathrm{n}_{\mathrm{u}}=$ $0.48+\mathrm{i} 2.45$ and $\mathrm{n}_{\mathrm{w}}=3.5+\mathrm{i} 2.73$ corresponding to $\mathrm{a}$ wavelength equal $0.55 \mu \mathrm{m}$.

\subsection{Rectangular Surfaces}

For an angle of incidence equal to $1^{\circ}$, it follows an agreement between the differential method (CWA) and the geometric method (GOA) for gold and tungsten in the following cases: From the ratio $d / \lambda$ equal to 1.6 when the height $\mathrm{h}$ of the grating is equal $0.1 \lambda$ (Figures $\mathbf{3}(\mathbf{a}, \mathbf{b}))$, and ratio $d / \lambda$ above 3.2 when $\mathrm{h}$ is equal $1 \lambda$ (Figures 3(c,d)). These two limits can be translated respectively by angles $\beta$ lower to $7.12^{\circ}$ and $32^{\circ}$.

For height $\mathrm{h}$ equal $0.1 \lambda$, the asymptotic limit of emissivity curves, reflecting the agreement between the CWA and GOA corresponds to the values 0.21 and 0.51 in the case of gold and tungsten respectively [26]. These limits are those of the emissivity of the smooth surface in the direction 1, calculated using the Fresnel formulas [26-30].

In these conditions $\left(h=0.1 \lambda, \theta=1^{\circ}\right)$, the reflection is simple at any point in the grating; the agreement between the two methods is possible only for a period $d$ exceeding $1.6 \lambda$. It is thus clear that the validity of GOA is not exclusively related to the number of reflections within the groove. However, it is remarkably to note that the GOA is valid for grating for height equal $1 / 10 \lambda$ and period equal $\lambda$. For the same angle of incidence $1^{\circ}$, and the same reports $d / \lambda$ from 0.05 to 12.08 ,

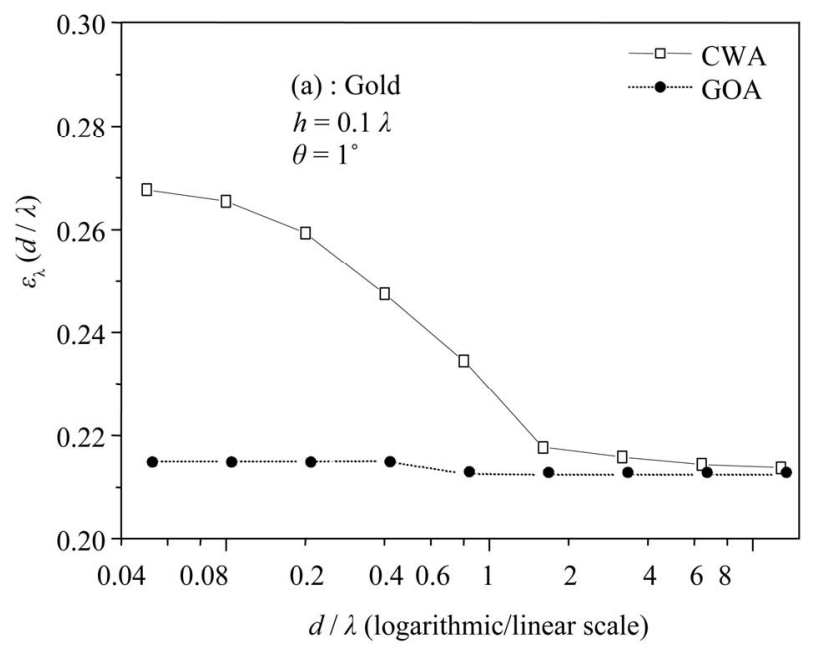

(a)

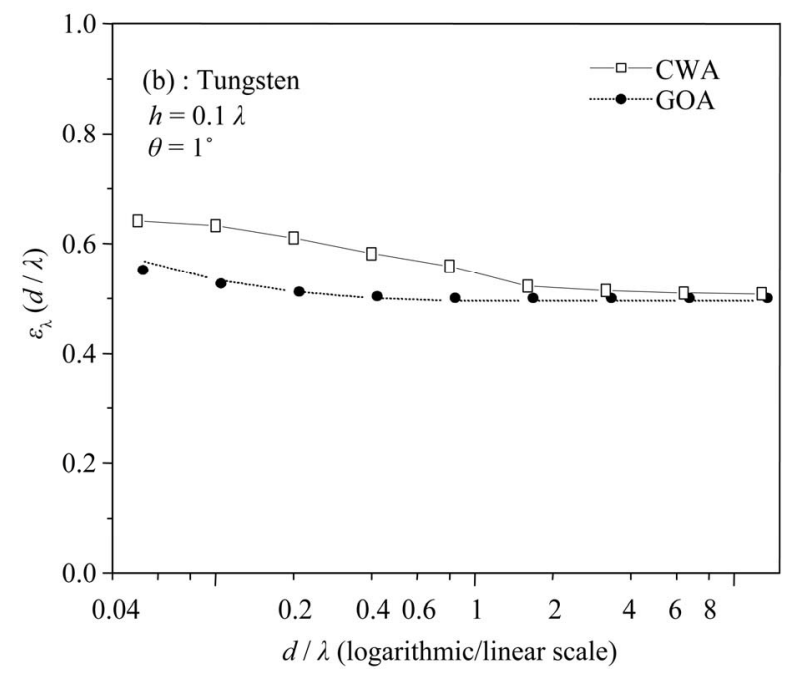

(b)

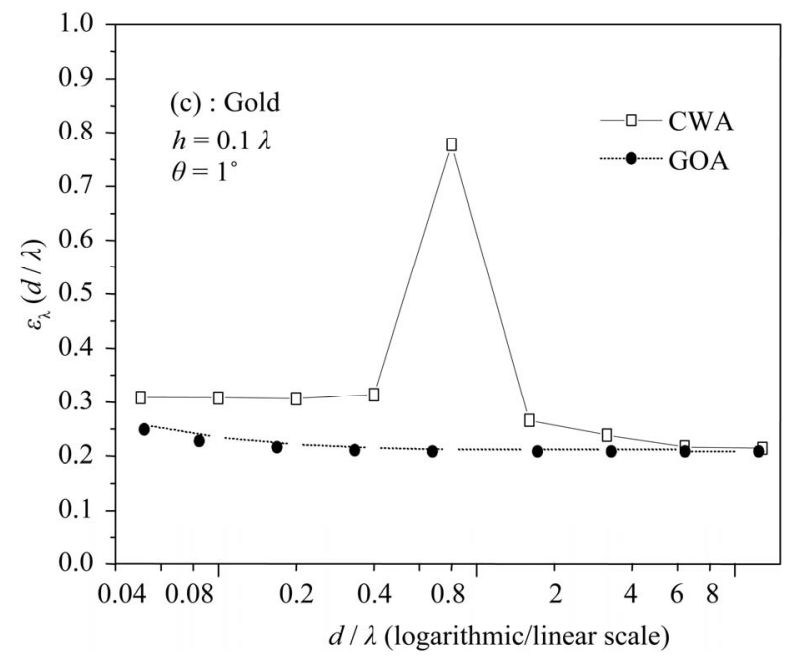

(c)

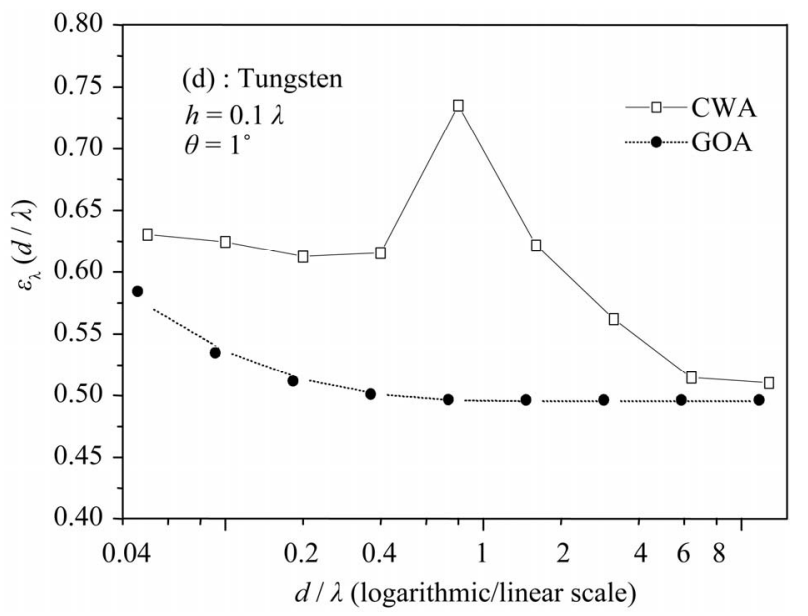

(d)

Figure 3. Comparison of differential method (CWA) with geometric optics approximation (GOA) for the directional monochromatic emissivity $\varepsilon_{\lambda}(d / \lambda)$ of rectangular surfaces. 
we clearly remark that the validity domain of GOA increases dramatically when the height increased from $0.1 \lambda$ to $1 \lambda$. In terms of angle $\beta$ characteristic of the cavity the domain of validity is from $\left[0^{\circ}, 7^{\circ}\right]$ to $\left[0^{\circ}, 32^{\circ}\right]$.

In our study, it is important to note that for a groove height $1 \lambda$, it suffices that the period exceeds $3.2 \lambda$ for the GOA to become valid. In the case of figures (Figures $3(\mathbf{c}, \mathbf{d})$ ), reflection on the inside of the groove is easy, especially since the direction of incidence very close to normal there is no shadow effect.

\subsection{Sinusoidal Surfaces}

Emissivity curves $\varepsilon_{\lambda}(d / \lambda)$, for both h and $\theta$ fixed, calculated by the two methods (Figures $4(\mathbf{a}, \mathbf{b})$ ) to determine the value of the ratio limit $(d / \lambda)_{\text {lim }}$ from which the GOA is valid. We associate the validity domain of GOA to the phenomenon of multiple reflection inside the cavity and that of the shadow effect due to the surface $[27,28]$.

This effect is negligible for directions of incidence around normal $\left(\theta=1^{\circ}\right.$ ) (Figure 4(a)) because to consider only simple reflection. For directions $\left(\theta=60^{\circ}\right)$ (Figure 4(b)), the shading effect is enhanced and this effect is also associated with the phenomenon of multiple reflections.

\subsection{The Range of Validity of the GOA}

We consider the previous study in the case of gold for heights $\mathrm{h}$ and for incidence angles from $10^{\circ}$ to $80^{\circ}$ with a step of $10^{\circ}$. In this report limit $(d / \lambda)_{\lim }$ is associated, for $\mathrm{h}$ fixed, the limiting slope $(2 h / d)$ lim. Figure 5 shows curves the limiting slope versus the cosine of the angle of incidence or emission $\theta$, for a fixed height $h$. This curve defines two regions. For the first, situated

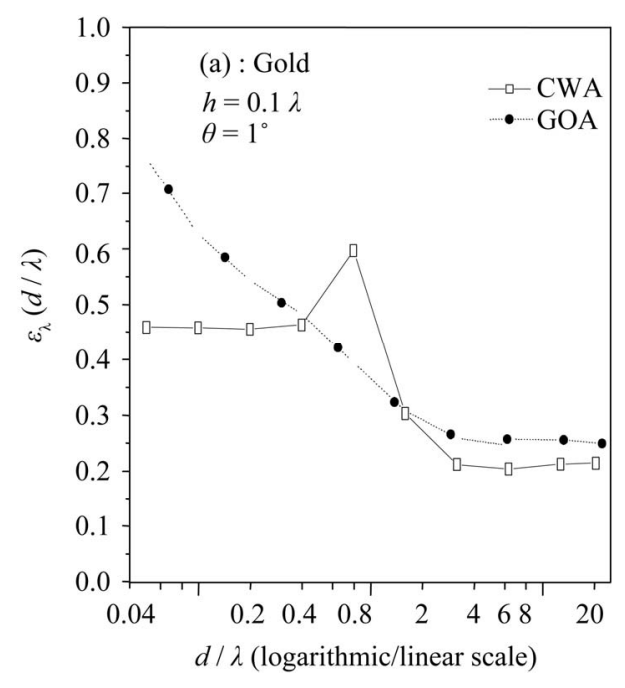

(a)

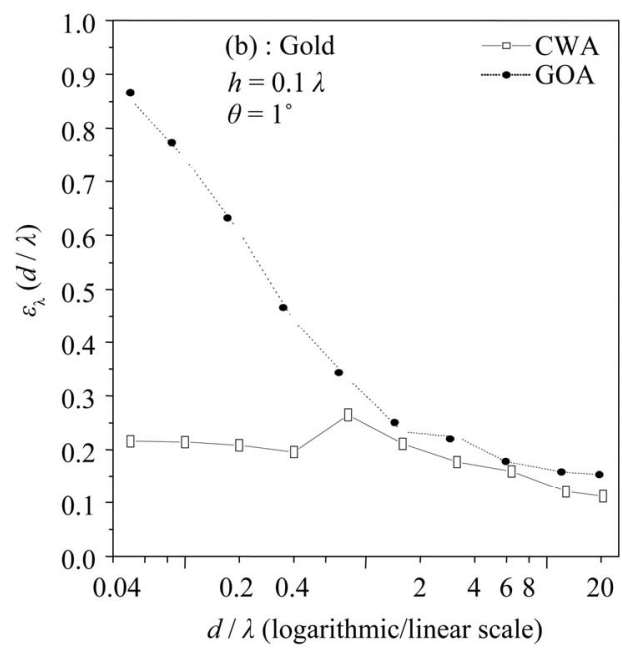

(b)

Figure 4. Comparison of differential method (CWA) with geometric optics approximation (GOA) for the directional monochromatic emissivity $\varepsilon_{\lambda}(d / \lambda)$ of sinusoidal surfaces.

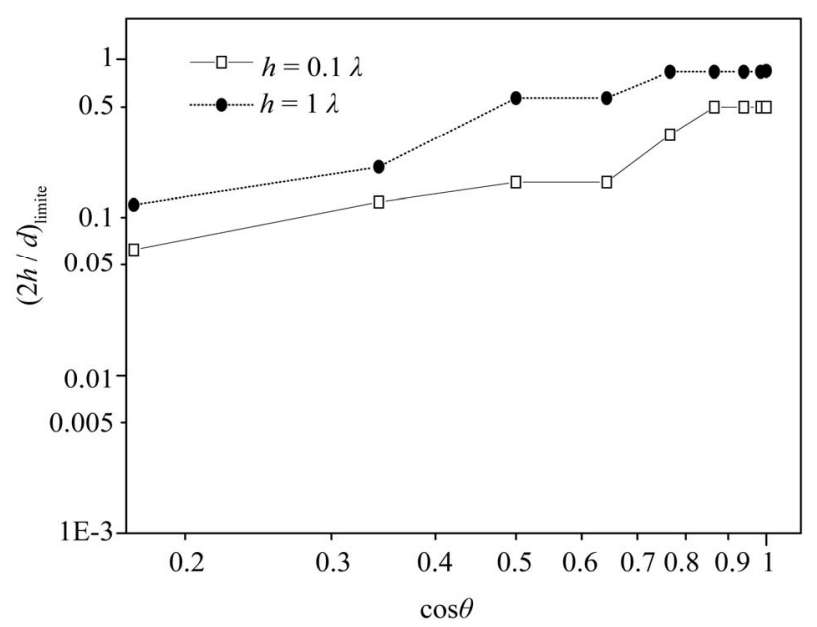

Figure 5. Differential method of sinusoidal surfaces domain plot with region validity for the geometric optics approximation in terms of limiting slope $(2 h / d)_{\lim }$.

above this curve, using the CWA or another accurate method is necessary since GOA is not valid. This is very satisfactory at all points of the second region located below the same curve.

By adopting the previous approach, for $h / \lambda$ and $\theta$ fixed, by varying the ratio $d / \lambda$, we first determine the limits of validity of GOA compared with CWA respectively, for incidence angles 1 and $60^{\circ}$ and reports $h / \lambda$ going up to value 10 with a step equal to 2 . We include the points determined from the curves of Figure 5. Thus, Figure 6 gives a significant idea about the range of the geometrical optics approximation validity. In fact under the cloud of points in this figure the approximation of 
geometrical optics is valid, so that above the same cloud the use of an exact method is necessary.

\section{HOMOGENIZATION REGIME}

We analyze the behaviour of emissivity when the period is very small compared to the wavelength. Under these conditions the grating is equivalent to a superposition of layers of effective indices determined using the effective medium theory [26]. We compare the emissivities calculated by the homogenization process, for $d / \lambda$ equal to 0.05 , in the cases discussed above with that calculated by the CWA and GOA. We see a very good agreement with the differential method in all these cases, however no agreement with the method of geometrical optics can be reported according to what we should expect (Tables 1,2).

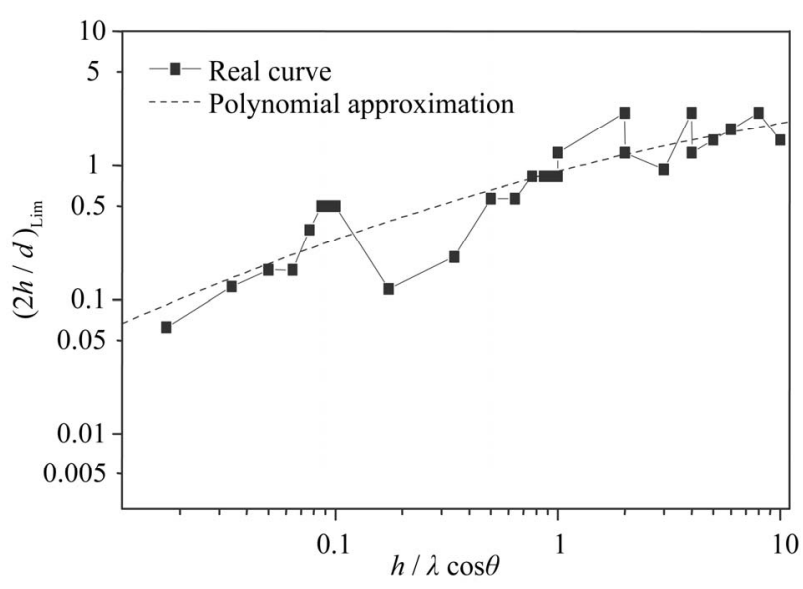

Figure 6. Ratio $(2 h / d)_{\lim }$ as function of $(h / \lambda) \cos \theta$.

Table 1. (a) and (b): Comparison between the emissivity calculated by the homogenization process and those calculated by the methods of differential and geometrical optics for $d / \lambda$ equal to 0.05 , in the case of TE polarization. Case of gold and tungsten - rectangular surfaces.

(a)

\begin{tabular}{ccccc}
\hline $\mathrm{h}$ & $\theta$ & CWA (Gold) & Homogenization (Gold) & GOA (Gold) \\
\hline $0.1 \lambda$ & $1^{\circ}$ & 0.2612 & 0.2681 & 0.2574 \\
$0.1 \lambda$ & $60^{\circ}$ & 0.1313 & 0.1346 & 0.5399 \\
$1 \lambda$ & $1^{\circ}$ & 0.3066 & 0.3082 & 0.2589 \\
$1 \lambda$ & $60^{\circ}$ & 0.1497 & 0.1505 & 0.5409 \\
\hline
\end{tabular}

(b)

\begin{tabular}{ccccc}
\hline $\mathrm{h}$ & $\theta$ & $\begin{array}{c}\text { CWA } \\
\text { (Tungsten) }\end{array}$ & $\begin{array}{c}\text { Homogenization } \\
\text { (Tungsten) }\end{array}$ & $\begin{array}{c}\text { GOA } \\
\text { (Tungsten) }\end{array}$ \\
\hline $0.1 \lambda$ & $1^{\circ}$ & 0.6381 & 0.6443 & 0.4261 \\
$0.1 \lambda$ & $60^{\circ}$ & 0.3942 & 0.3997 & 0.5409 \\
$1 \lambda$ & $1^{\circ}$ & 0.6281 & 0.6320 & 0.4284 \\
$1 \lambda$ & $60^{\circ}$ & 0.3880 & 0.3913 & 0.5409 \\
\hline
\end{tabular}

Table 2. (a) and (b): Comparison between the emissivity calculated by the homogenization process and those calculated by the methods of differential and geometrical optics for $d / \lambda$ equal to 0.05 , in the case of TE polarization. Case of gold and tungsten - rectangular surfaces.

(a)

\begin{tabular}{ccccc}
\hline $\mathrm{h}$ & $\theta$ & CWA(Gold) & Homogenization (Gold) & GOA (Gold) \\
\hline $0.1 \lambda$ & $1^{\circ}$ & 0.2509 & 0.2520 & 0.4712 \\
$0.1 \lambda$ & $60^{\circ}$ & 0.1267 & 0.1273 & 0.5846 \\
$1 \lambda$ & $1^{\circ}$ & 0.4586 & 0.4620 & 0.7650 \\
$1 \lambda$ & $60^{\circ}$ & 0.2156 & 0.2177 & 0.8547 \\
\hline
\end{tabular}

(b)

\begin{tabular}{ccccc}
\hline $\mathrm{h}$ & $\theta$ & $\begin{array}{c}\text { CWA } \\
\text { (Tungsten) }\end{array}$ & $\begin{array}{c}\text { Homogenization } \\
\text { (Tungsten) }\end{array}$ & $\begin{array}{c}\text { GOA } \\
\text { (Tungsten) }\end{array}$ \\
\hline $0.1 \lambda$ & $1^{\circ}$ & 0.6396 & 0.6449 & 0.7624 \\
$0.1 \lambda$ & $60^{\circ}$ & 0.3953 & 0.3995 & 0.6747 \\
$1 \lambda$ & $1^{\circ}$ & 0.8228 & 0.8201 & 0.9319 \\
$1 \lambda$ & $60^{\circ}$ & 0.5670 & 0.5754 & 0.9351 \\
\hline
\end{tabular}

\section{CONCLUSIONS}

In this paper, we study the validity limits of the of geometrical optics approximation compared with a differential method. We determine by both methods the emissivity for rectangular and sinusoidal surfaces of gold and tungsten, when the wavelength is equal to 0.55 microns.

The results obtained by the exploitation of the codes elaborated for the three methods and for TE polarization are validated. The obtained results and the presented interpretations lead to the following conclusions:

In case of an angle of incidence equal to $1^{\circ}$ and a height of roughness equal $0.1 \lambda$, we find an agreement between the differential method and the approximation of geometrical optics as well as for gold and tungsten for a period greater than $1.6 \lambda$.

For the same incidence angle $1^{\circ}$, and the same reports $d / \lambda$, we conclude that the validity domain of GOA increases when the height of the roughness increases from $0.1 \lambda$ to $1 \lambda$.

In the case of an angle of incidence equal to $60^{\circ}$ and for both materials, the agreement between the two methods is obtained as soon as the ratio $d / \lambda$ becomes greater than 0.8 .

The simple reflection condition for the GOA is necessary but not sufficient.

For $h=1 \lambda$ the shadowing phenomenon does not prohibit the validity of the geometric method.

The emissivities obtained by the homogenization method are in perfect agreement with those calculated by the differential method when the period of the profiles is very small compared to the wavelength. 


\section{REFERENCES}

[1] Carminati, R. and Greffet, J.J. (1998) A model for the radiative properties of opaque rough surfaces. Heat transfer, Proceedings of 11th IHTC, 7, Kyongyu, Koria, 23-28 August 1998.

[2] Le, G., Olivier, J. and Greffet, J.J. (1997) Experimental and theoretical, porting a surface-phonon polariton. Physical Review B, 55, 10105-10114. doi:10.1103/PhysRevB.55.10105

[3] Chinnok, C. (1994) Laser based systems easily find semiconductor defects. Laser Focus World, 30, 59-63.

[4] He, X., Torranse, Sillon, F.X. and Greenberg, D. (1991) A comprehensive physical model for light reflection. Computer Graphics, Annual Conference Serie, 25, 175186.

[5] Kakeun, T., Dimenna, R.A. and Buckius, R. (1997). Region of validity of the geometric optics approximation for angular scattering from very rough surfaces. International Journal of Heat and Mass Transfer, 40, 49-59.

[6] Ranches, G.J.A. and Vesperinas, M.N. (1991) Light scattering from random rough dielectric surfaces. The Journal of the Optical Society of America, 8, 1270-1286.

[7] Kong, J.A. (1977) Second-order coupled-mode equations for spatially periodic media. Journal Optical Society of America, 67, 825-829. doi.: 10.1364/JOSA.67.000825

[8] Moharam, M.G. and Gaylord, T.K. (1980) Criteria for bragg regime diffraction by phase gratings. Optics Communications, 32, 14-22.

doi:10.1016/0030-4018(80)90304-1

[9] Moharam, M.G. and Gaylord, T.K. (1981) Coupled-wave analysis of reflection gratings. Applied Optics, 20, 240-244.

[10] Moharam, M.G. and Gaylord, T.K. (1981) Rigorous coupled-wave analysis of planar grating diffraction. The Journal of the Optical Society of America, 71, 811-818. doi:10.1364/JOSA.71.000811

[11] Moharam, M.G. and Gaylord, T.K. (1982) Chain-matrix analysis of arbitrary-thickness dielectric reflection gratings. Journal Optical Society of America, 72, 187-189. doi:10.1364/JOSA.72.000187

[12] Moharam, M.G. and Gaylord, T.K. (1983) Rigorous coupled-wave analysis of grating diffraction-E-mode polarization and losses. Journal Optical Society of America, 73, 451-455. doi:10.1364/JOSA.73.000451

[13] Moharam, M.G. and Gaylord, T.K. (1982). Diffraction analysis of dielectric surface-relief gratings. Journal Optical Society of America, 72, 1385-1392. doi:10.1364/JOSA.72.001385

[14] Moharam, M.G. and Gaylord, T.K. (1986) Rigorous coupled-wave analysis of metallic surface-relief gratings. Journal Optical Society of America. A, 1, 1780-1787.

[15] Chateau, N. (1994) Algorithm for the rigorous coupled-wave analysis of grating diffraction. Journal Optical Society of America. A, 11, 1321-1331.

[16] Pai, D.M. and Awada, K.A. (1991) Analysis of dielectric gratings of arbitrary profiles and thicknesses. Journal Optical Society of America. A, 8, 755-762.
[17] Li, L.F. and Haggans, C.W. (1993) Convergence of the coupled-wave method for metallic lamellar diffraction gratings. Journal Optical Society of America. A, 10, 1184-1189. doi:10.1364/JOSAA.10.001184

[18] Ody Sacadura, J.F. (1972) Influence de la rugosité sur le rayonnement thermique émis par les surfaces opaques, Essai de modèle. Journal Int. Heat Mass Transfer, 15, 1451-1465.

[19] Kanayama, K. (1972) Apparent directionnal emitances of $\mathrm{V}$ groove and circulair-groove rough surfaces. Journal Heat Transfer Japenese Research, 1, 11-32.

[20] Druelle, P. (1978) Influence de macro-rugosités sur les caractéristiques radiatives de certaines surfaces. Application aux acier inoxydables, Thesis of Doctor Engineer. Central School of Arts and Manufactures.

[21] Druelle, P. (1980) Caractéristiques radiatives des aciers inoxydables dans le domaine du visible, Schématisation de la rugosité à l'aide de calottes sphériques et étude d'un capteur solaire hémisphérique, Thesis of Doctor Engineer. Central School of Arts and Manufactures.

[22] Ghamari, F. (1996) Contribution à l'étude de l'influence de la rugosité sur l'émissivité des surfaces opaques, PHD Thesis, Faculty of Sciences Tunis.

[23] Bouchitté, R. and Petit, R. (1985) Homogeinization techniques as applied in the electromagnetic theory of gratings. Electromagnetics, 5, 17-36. doi:10.1080/02726348508908135

[24] Southwell, W.H. (1991) Pyramid-array surface-relief structures proceducing antireflection index matching on optical surfaces. Journal Optical Society of America. A, 8, 549-553. doi:10.1364/JOSAA.8.000549

[25] Petit, R. and Vincent, P. (1980) Electromagnetic theory of gratings. Springer-Verlag, Berlin, Heidelberg, New York.

[26] Ghabara, T. (2003) Etude de l'emissivite des surfaces rugueuses periodiques a l'aide des methods d'analyse en ondes couplees. Comparaison avec la methode de l'optique geometrique. PHD Thesis, Faculty of Sciences Tunis.

[27] Ghamari, F. and Ghabara, T. (2004) Influence of microroughness on emissivity. Journal of Applied Physics, 96, 2656-2664. doi:10.1063/1.1776634

[28] Ghabara, T., Ghmari, F. and Sifaoui, S. (2007) Emissivity of triangular surfaces determined by differential method: From homogenization to validity limit of geometrical optics. American Journal of Applied Sciences, 4, 146-154. doi:10.3844/ajassp.2007.146.154

[29] Ghamari, F., Sassi, I. and Sifaoui, M. (2005) Directional hemispherical radiative properties of random dielectric rough surfaces. Waves in Random and Complex Media, 15, 469. doi: $10.1080 / 17455030500361838$

[30] Matchiane, M.B., Ghamari, F. and Sifaoui (2004) Correction de L'approximation de Kirchhoff par la methode integrale reformulee, cas des reflectivites de surfaces sinusoidale. Canadian Journal of Physics, 82, 303. doi:10.1139/p04-008

[31] Sassi, I. and Sifaoui, M.S. (2007) Comparison of geometric optics approximation and integral method for reflection and transmission from microgeometrical dielec- 
tric surfaces. American Journal of Applied Sciences, 4, 451-462.

[32] Sassi, I. and Sifaoui, M.S. (2009) Effect of the material of rough surfaces and the incident light polarization on the validity of the surface impedance boundary condition and the geometric optics approximation for reflection and emission. American Journal of Applied Sciences, 4, 480488. 